\title{
Finite-time synchronization of drive-response systems via periodically intermittent adaptive control
}

\author{
Jun Mei ${ }^{a, b}$, Minghui Jiang ${ }^{a, *}$, Xiaohong Wang ${ }^{c}$, Jiali Han ${ }^{a}$, Shuangtao Wang ${ }^{a}$ \\ ${ }^{a}$ College of Science, China Three Gorges University, Yichang, Hubei 443002, China \\ ${ }^{b}$ Centre of New Energy Systems, Department of Electrical, Electric and Computer Engineering, \\ University of Pretoria Pretoria 0002, South Africa \\ ${ }^{c}$ College of Information Engineering, Henan University of Science and Technology, \\ Luoyang 471023, China
}

\begin{abstract}
In this paper, the finite-time synchronization between two complex dynamical networks via the periodically intermittent adaptive control and periodically intermittent feedback control is studied. The finite-time synchronization criteria are derived based on finite-time stability theory, the differential inequality and the analysis technique. Since the traditional synchronization criteria for some models are improved in the convergence time by using the novel periodically intermittent adaptive control and periodically intermittent feedback control, the results of this paper are important. Numerical examples are finally presented to illustrate the effectiveness and correctness of the theoretical results.
\end{abstract}

Keywords: Drive-response systems; finite-time synchronization; periodically intermittent adaptive control; periodically intermittent feedback control.

\section{Introduction}

For the last decade or so, models of complex networks have been widely used to research real systems in nature, society and engineering. A complex network consists of a large set of interconnected nodes, where a node is a fundamental unit with detailed contents. These nodes may have different meanings in different situations, such as microprocessors, computers, companies, and so on. However, the network structure facilitates and constrains the network dynamical behaviors.

In the past decades, the control and synchronization of complex dynamical networks has attracted much attention and some relevant theoretical results have been established [1-7],

* Corresponding author.

E-mail address: meij0000@163.com (J. Mei), *jiangminghui@ctgu.edu.cn (M. Jiang), wxhong2006@163.com (X. Wang). 
due to its many potential practical applications. Many effective control methods including adaptive control [8-14], feedback control [15-18], observer control [19-20], pinning control [2123], impulsive control [24-28] and intermittent control [29-37] have been proposed to drive the network to achieve synchronization. Among these control approaches, the discontinuous control methods which include impulsive control and intermittent control have attracted much interest due to its practical and easy implementation in engineering fields. But, the intermittent control is different from the impulsive control since impulsive control is activated only at some isolated instants, while intermittent control has a nonzero control width, the explain can be found in the map (see Fig. 1). Obviously, when $u=v$, the intermittent control becomes the general continuous control, while $u=0$, the intermittent control becomes the impulsive control. Besides, using intermittent control is more effective and robust [38]. Therefore, in recent years, many synchronization criteria for complex dynamical networks with or without time delays via intermittent control have been presented, see [30-31, 39-40].

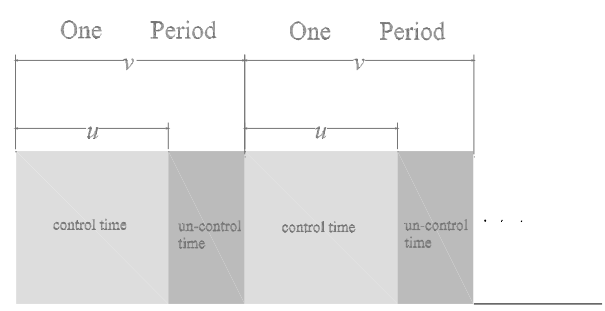

Fig.1. Sketch map of the intermittent control, i.e., the control time is periodic, and in any period, the time is composed of "control time" and "un-control time".

Recently, most of the current researches were primarily concerned with asymptotical or exponential synchronization of networks via intermittent control [29-31]. This indicates that the intermittent control can derive the slave system to synchronization the master system after the infinite horizon. However, in reality, the networks might always be expected to achieve synchronization or stability under a finite time, particularly in engineering fields. In order to achieve synchronization in a given time, the useful and efficient intermittent control methods might be finite-time intermittent control. Finite-time synchronization of complex networks via intermittent control means the optimality in convergence time dealing with intermittent control problems. However, to our best knowledge, there is few published 
paper considering finite-time synchronization of complex dynamical networks via periodically intermittent control. Considering the important role of synchronization of complex networks, it is worth studying the finite-time synchronization of complex dynamical networks with intermittent control.

The main contribution of this paper lies in the following aspects. Firstly, a central lemma is proved by using analysis method. Additionally, two different intermittent controllers are designed to synchronize the addressed complex networks and some useful finite-time criteria are obtained. Subsequently, some sufficient conditions are also derived in terms of linear matrix inequality, which is very easy to verify. Besides, a very interesting fact is revealed that the more the control gains are, the longer in time to achieve finite-time synchronization will be.

The rest of this paper is organized as follows. In Section 2, the synchronization problem of a general complex network is formulated, and some useful lemmas and preliminaries are given. Finite-time synchronization of two complex dynamical networks by intermittent adaptive control theory is rigorously derived in Section 3. Section 4 studies the finitetime synchronization of drive-response networks by using the intermittent updated laws of coupling strengths. Some numerical examples are given for supporting the theory results in Section 5. Conclusions are drawn in Section 6.

\section{Preliminaries}

In this paper, we firstly introduce a complex network model considered in our work and give some useful mathematical preliminaries.

Consider a complex dynamical network consisting of $N$ nodes, in which each node is an n-dimensional dynamical system. The state equation of the entire network is given as:

$$
\dot{x}_{i}(t)=f\left(x_{i}(t)\right)+c \sum_{j=1}^{N} a_{i j} \Gamma x_{j}(t), \quad i=1,2, \cdots, N,
$$

where $x_{i}(t)=\left(x_{i 1}(t), x_{i 2}(t), \cdots, x_{i n}(t)\right)^{T} \in R^{n}$ is the state vector of the $i$ th dynamical node, $f: R^{n} \rightarrow R^{n}$ standing for the activity of an individual subsystem is a vector value function, the constant $c>0$ is a coupling strength. $\Gamma \in R^{n \times n}$ is a positive definite matrix which describe the individual coupling between node $i$ and node $j$ and $A=\left(a_{i j}\right) \in R^{N \times N}$ is the coupling configuration matrix. If there is a connection from the nodes $i$ to $j(j \neq i)$, then $a_{i j}>0$; otherwise, $a_{i j}=0(j \neq i)$, and the diagonal elements of matrices $A$ is defined as

$$
a_{i i}=-\sum_{j=1, j \neq i}^{N} a_{i j} .
$$

Throughout this paper, we have the following assumption. 
Assumption 1. ([44]) Assume that there exists a positive definite diagonal matrix $P=$ $\operatorname{diag}\left(p_{1}, \ldots, p_{n}\right)$ and a diagonal matrix $\Theta=\operatorname{diag}\left(\theta_{1}, \ldots, \theta_{n}\right)$, such that $f(\cdot)$ satisfies the following inequality:

$$
(y-x)^{T} P(f(y)-f(x)-\Theta(y-x)) \leq-\xi(y-x)^{T}(y-x),
$$

for some $\xi>0$, all $x, y \in R^{n}$ and $t>0$.

We can obtain that many well-known chaotic systems such as the Chua's oscillators, Rössler systems, Lorenz systems, Chen systems, and Lü systems satisfy Assumption 1.

Lemma 1 ([41]). Assume that a continuous, positive-definite function $V(t)$ satisfies the following inequality:

$$
\dot{V}(t) \leq-\alpha V^{\eta}(t), \quad \forall t \geq t_{0}, \quad V\left(t_{0}\right) \geq 0,
$$

where $\alpha>0,0<\eta<1$ are two constants. Then, for any given $t_{0}, V(t)$ satisfies the following inequality:

$$
V^{1-\eta}(t) \leq V^{1-\eta}\left(t_{0}\right)-\alpha(1-\eta)\left(t-t_{0}\right), t_{0} \leq t \leq t_{1},
$$

and

$$
V(t) \equiv 0, \quad \forall t \geq t_{1},
$$

with $t_{1}$ given by

$$
t_{1}=t_{0}+\frac{V^{1-\eta}\left(t_{0}\right)}{\alpha(1-\eta)} .
$$

Lemma 2 ([48]). Let $x_{1}, x_{2}, \cdots, x_{n} \in R^{m}$ are any vectors and $0<q<2$ is a real number satisfying:

$$
\left\|x_{1}\right\|^{q}+\left\|x_{2}\right\|^{q}+\cdots+\left\|x_{n}\right\|^{q} \geq\left(\left\|x_{1}\right\|^{2}+\left\|x_{2}\right\|^{2}+\cdots+\left\|x_{n}\right\|^{2}\right)^{q / 2} .
$$

Lemma 3. Suppose that function $V(t)$ is continuous and non-negative when $t \in[0, \infty)$ and satisfies the following conditions:

$$
\begin{cases}\dot{V}(t) \leq-\alpha V^{\eta}(t), & l T \leq t \leq l T+\theta T \\ \dot{V}(t) \leq 0, & l T+\theta T \leq t<(l+1) T\end{cases}
$$

where $\alpha>0, T>0,0<\eta, \theta<1, l \in \ell=\{0,1,2, \ldots, p\}$ is a finite natural number set and $p$ is a positive integer, then the following inequality holds:

$$
V^{1-\eta}(t) \leq V^{1-\eta}(0)-\alpha \theta(1-\eta) t, \quad 0 \leq t \leq \bar{T},
$$

for the constant $\bar{T}$ is the setting time.

Proof. Take $M_{0}=V^{1-\eta}(0)$ and $W(t)=V^{1-\eta}(t)+\alpha(1-\eta) t$, where $t \geq 0$. Let $Q(t)=$ $W(t)-h M_{0}$, where $h>1$ is a constant. It is easy to see that

$$
Q(t)<0, \quad \text { for } t=0 \text {. }
$$


Next, we will prove that

$$
Q(t)<0, \quad \text { for all } t \in[0, \theta T) .
$$

Otherwise, there exists a $t_{0} \in[0, \theta T)$ such that

$$
\begin{array}{ll}
Q\left(t_{0}\right)=0, & \dot{Q}\left(t_{0}\right)>0, \\
Q(t)<0, & 0 \leq t<t_{0} .
\end{array}
$$

Using Eqs. (2.6), (2.8) and (2.9), we obtain

$$
\begin{aligned}
\dot{Q}\left(t_{0}\right) & =(1-\eta) V^{-\eta}(t) \dot{V}(t)+\alpha(1-\eta) \\
& \leq(1-\eta) V^{-\eta}(t)\left(-\alpha V^{\eta}(t)\right)+\alpha(1-\eta) \\
& =-\alpha(1-\eta)+\alpha(1-\eta)=0
\end{aligned}
$$

This contradicts the second inequality in (2.8), and so (2.7) holds.

Now, we prove that for $t \in[\theta T, T)$

$$
H(t)=W(t)-h M_{0}-\alpha(1-\eta)(t-\theta T)<0 .
$$

Otherwise, there exists a $t_{1} \in[\theta T, T)$ such that

$$
\begin{array}{ll}
H\left(t_{1}\right)=0, & \dot{H}\left(t_{1}\right)>0, \\
H(t)<0, & \theta T \leq t<t_{1} .
\end{array}
$$

Using Eqs. (2.12) and (2.13), we have

$$
\begin{aligned}
\dot{H}\left(t_{1}\right) & =(1-\eta) V^{-\eta}\left(t_{1}\right) \dot{V}\left(t_{1}\right)+\alpha(1-\eta)-\alpha(1-\eta) \\
& \leq \alpha(1-\eta)-\alpha(1-\eta) \\
& =0
\end{aligned}
$$

which contradicts the second inequality in (2.12). Hence (2.11) holds.

Consequently, on the one hand, for $t \in[\theta T, T)$,

$$
W(t)<h M_{0}+\alpha(1-\eta)(t-\theta T) \leq h M_{0}+\alpha(1-\eta)(1-\theta) T .
$$

On the other hand, it follows from Eqs. (2.6) and (2.7) that for $t \in[0, \theta T)$

$$
W(t)<h M_{0}<h M_{0}+\alpha(1-\eta)(1-\theta) T .
$$

So

$$
W(t)<h M_{0}+\alpha(1-\eta)(1-\theta) T, \quad \text { for all } t \in[0, T) .
$$


Similarly, we can prove that for $t \in[T,(1+\theta) T)$,

$$
W(t)<h M_{0}+\alpha(1-\eta)(1-\theta) T
$$

and for $t \in[(1+\theta) T, 2 T)$

$$
\begin{aligned}
W(t) & <h M_{0}+\alpha(1-\eta)(1-\theta) T+\alpha(1-\eta)(t-\theta T-T) \\
& =h M_{0}+\alpha(1-\eta)(t-2 \theta T) .
\end{aligned}
$$

In the following, we can derive the following estimation of $W(t)$ for any integers $l$.

For $l T \leq t<(l+\theta) T$,

$$
W(t)<h M_{0}+\alpha(1-\eta)(1-\theta) l T,
$$

and for $(l+\theta) T \leq t<(l+1) T$,

$$
W(t)<h M_{0}+\alpha(1-\eta)[t-(l+1) \theta T] .
$$

Since for any $t \geq 0$, there exists a natural integer $k \in \ell$, such that $k T \leq t<(k+1) T$, by induction, we can conclude the following estimation of $W(t)$ for any $t$ by Eqs. (2.15) and (2.16)

For $k T \leq t<(k+\theta) T$,

$$
W(t)<h M_{0}+\alpha(1-\eta)(1-\theta) k T \leq h M_{0}+\alpha(1-\eta)(1-\theta) t,
$$

and for $(k+\theta) T \leq t<(k+1) T$,

$$
W(t)<h M_{0}+\alpha(1-\eta)[t-(k+1) \theta T] \leq h M_{0}+\alpha(1-\eta)(1-\theta) t .
$$

Let $h \rightarrow 1$, from the definition of $W(t)$, we ontain

$$
V^{1-\eta}(t) \leq V^{1-\eta}(0)-\alpha(1-\eta) t+\alpha(1-\eta)(1-\theta) t=V^{1-\eta}(0)-\alpha \theta(1-\eta) t, \quad t \geq 0 .
$$

Lemma 4. When $a \leq b$ and $c<1$ are all positive numbers, then the following inequality holds:

$$
a^{c} \leq b^{c} .
$$

The proof is easily obtained, and this proofs is omitted.

\section{Finite-time synchronization of complex networks via intermittent adaptive control}


In this section, we consider the finite-time synchronization of general complex dynamical networks via periodically intermittent control.

For simplicity, regarding model (2.1) as the master (or drive) system, and the response (or slave) system is given by:

$$
\dot{y}_{i}(t)=f\left(y_{i}(t)\right)+c \sum_{j=1}^{N} a_{i j} \Gamma y_{j}(t)+u_{i}(t), \quad i=1,2, \cdots, N,
$$

where $y_{i}(t)=\left(y_{i 1}(t), y_{i 2}, \ldots, y_{i n}(t)\right)^{T} \in R^{n}$ is the response state vector of the node $i . u(t)=$ $\left(u_{1}(t), u_{2}(t), \ldots, u_{N}(t)\right)^{T}$ is an intermittent controller defined by

$$
\left\{\begin{array}{l}
u_{i}(t)=r_{i} e_{i}-\bar{k} \frac{\sqrt{\lambda_{\max }(P)}}{{ }^{\lambda} \min ^{(P)}} \operatorname{sign}\left(e_{i}\right), l T \leq t<l T+\delta, \\
u_{i}(t)=0, \quad\left\|e_{i}\right\|=0 \text { or } l T+\delta \leq t<(l+1) T,
\end{array}\right.
$$

where $r_{i}(i=1,2, \ldots, N)$ are the adaptive update laws, $\operatorname{sign}(\cdot)$ function $\operatorname{sign}\left(e_{i}\right)=\left(\operatorname{sign}\left(e_{i 1}\right)\right.$, $\left.\operatorname{sign}\left(e_{i 2}\right), \cdots, \operatorname{sign}\left(e_{i n}\right)\right)^{T} . l \in \ell=\{0,1,2, \ldots, p\}$ is a finite natural number set and $p$ is a positive integer, $\bar{k}>0$ is a tunable constant and real number, $T>0$ is the control period, $\delta>0$ is called the control width (control duration). $P$ is a positive definite diagonal matrix. Denote $\lambda_{\max }(P)\left(\lambda_{\min }(P)\right)$ as the maximum (minimum) eigenvalue of the positive definite diagonal matrix $P$.

Assume that $x(t)=\left(x_{1}(t), \ldots, x_{N}(t)\right)^{T}$ and $y(t)=\left(y_{1}(t), \ldots, y_{N}(t)\right)^{T}$ are solutions of system (2.1) and (3.1). Further, $e_{i}(t)=y_{i}(t)-x_{i}(t)$ be synchronization error between the states of drive system (2.1) and the response system (3.1), and $\theta=\delta / T$ be the ratio of the control width $\delta$ to the control period $T$ called control rate, then the error dynamical system can be derived as

$$
\left\{\begin{array}{l}
\dot{e}_{i}=f\left(y_{i}\right)-f\left(x_{i}\right)+c \sum_{j=1}^{N} a_{i j} \Gamma e_{j}+u_{i}, \\
l T \leq t<l T+\theta T, \quad i=1,2, \cdots, N \\
\dot{e}_{i}=f\left(y_{i}\right)-f\left(x_{i}\right)+c \sum_{j=1}^{N} a_{i j} \Gamma e_{j}, \\
l T+\theta T \leq t<(l+1) T, \quad i=1,2, \cdots, N
\end{array}\right.
$$

Meanwhile, the intermittent updated laws are taken as follows:

$$
\left\{\begin{array}{l}
\dot{r}_{i}=-\lambda_{i}\left[\frac{\eta_{i} r_{i} e_{i}^{T} P e_{i}}{r_{i}^{2}}+e_{i}^{T} P e_{i}+\frac{\bar{k}}{\sqrt{\lambda_{i}}} \operatorname{sign}\left(r_{i}\right)\right], l T \leq t<l T+\delta, \\
\dot{r}_{i}=0, \quad\left\|r_{i}\right\|=0 \text { or } l T+\delta \leq t<(l+1) T,
\end{array}\right.
$$

where $\eta_{i}>0(i=1,2, \ldots, N)$ is a positive constant called control gain, $\lambda_{i}>0$ is an arbitrary positive constant.

Then we can derive the following periodically intermittent synchronization criteria.

Theorem 1. Suppose Assumption 1 holds. If there exist a positive definite matrix $\Xi=$ 
$\operatorname{diag}\left(\eta_{1}, \eta_{2}, \ldots, \eta_{N}\right)>0$ and a positive definite diagonal matrix $P>0$ such that the following conditions hold:

$$
\begin{gathered}
\theta_{j} I_{N}-\Xi+c \gamma_{j} A \leq 0, \quad j=1,2, \ldots, n, \\
\theta_{j} I_{N}-\frac{\xi}{\lambda_{\max }(P)} I_{N}+c \gamma_{j} A \leq 0, \quad j=1,2, \ldots, n,
\end{gathered}
$$

where $\Gamma=\operatorname{diag}\left(\gamma_{1}, \gamma_{2}, \ldots, \gamma_{n}\right), \Theta=\operatorname{diag}\left(\theta_{1}, \theta_{2}, \ldots, \theta_{n}\right), \xi$ is a positive constant satisfies Assumption 1, and $I_{N}$ is an appropriate identity matrix. Then the error system (3.3) is synchronized under the periodically intermittent controller (3.2) and the intermittent updated law (3.4) in a finite time:

$$
T_{1}=\frac{2 V^{1 / 2}(0)}{\sqrt{2} \bar{k} \theta}
$$

where $V(0)=\frac{1}{2} \sum_{i=1}^{N} e_{i}^{T}(0) P e_{i}(0)+\frac{1}{2} \sum_{i=1}^{N} \frac{1}{\lambda_{i}}\left(r_{i}(0)\right)^{2}, e_{i}(0), r_{i}(0)$ are the initial conditions of $e_{i}, r_{i}$.

Proof. We design the following Lyapunov function:

$$
V(t)=\frac{1}{2} \sum_{i=1}^{N} e_{i}^{T} P e_{i}+\frac{1}{2} \sum_{i=1}^{N} \frac{1}{\lambda_{i}} r_{i}^{2} .
$$

Then the derivative of $V(t)$ with respect to time $t$ along the solutions of Eq. (3.3) can be calculated as follows:

When $l T \leq t<(l+\theta) T$, for $l \in \ell$,

$$
\begin{aligned}
\dot{V}(t)= & \sum_{i=1}^{N} e_{i}^{T} P \dot{e}_{i}+\sum_{i=1}^{N} \frac{1}{\lambda_{i}} r_{i} \dot{r}_{i} \\
= & \sum_{i=1}^{N} e_{i}^{T} P\left[f\left(y_{i}\right)-f\left(x_{i}\right)+c \sum_{j=1}^{N} a_{i j} \Gamma e_{j}+u_{i}\right]-\sum_{i=1}^{N} r_{i}\left[\frac{\eta_{i} r_{i} e_{i}^{T} P e_{i}}{r_{i}^{2}}+\right. \\
& \left.\frac{\bar{k}}{\sqrt{\lambda_{i}}} \operatorname{sign}\left(r_{i}\right)+e_{i}^{T} P e_{i}\right] \\
= & \sum_{i=1}^{N}\left\{e_{i}^{T} P\left[f\left(y_{i}\right)-f\left(x_{i}\right)-\Theta e_{i}\right]+e_{i}^{T} P \Theta e_{i}+e_{i}^{T} P u_{i}+\right. \\
& \left.c e_{i}^{T} P \sum_{j=1}^{N} a_{i j} \Gamma e_{j}\right\}-\sum_{i=1}^{N} r_{i}\left[\frac{\eta_{i} r_{i} e_{i}^{T} P e_{i}}{r_{i}^{2}}+\frac{\bar{k}}{\sqrt{\lambda_{i}}} \operatorname{sign}\left(r_{i}\right)+e_{i}^{T} P e_{i}\right] \\
\leq & -\xi \sum_{i=1}^{N} e_{i}^{T} e_{i}+\sum_{i=1}^{N} e_{i}^{T}\left(P \Theta-\eta_{i} P\right) e_{i}+c \sum_{i=1}^{N} e_{i}^{T} P \sum_{j=1}^{N} a_{i j} \Gamma e_{j} \\
& -\sum_{i=1}^{N} e_{i}^{T} P r_{i} e_{i}+\sum_{i=1}^{N} e_{i}^{T} P r_{i} e_{i}-\bar{k} \sum_{i=1}^{N} \frac{\sqrt{\lambda_{\max }(P)}}{\lambda_{\min }(P)} e_{i}^{T} P \operatorname{sign}\left(e_{i}\right) \\
& -\sum_{i=1}^{N} \frac{\bar{k}}{\sqrt{\lambda_{i}}}\left|r_{i}\right|
\end{aligned}
$$




$$
\begin{aligned}
\leq & \sum_{j=1}^{n} p_{j} \widetilde{e}_{j}^{T}\left(\theta_{j} I_{N}-\Xi+c \gamma_{j} A\right) \widetilde{e}_{j}-\bar{k} \sum_{i=1}^{N} \frac{\sqrt{\lambda_{\max }(P)}}{\lambda_{\min }(P)} e_{i}^{T} P \operatorname{sign}\left(e_{i}\right) \\
& -\sum_{i=1}^{N} \frac{\bar{k}}{\sqrt{\lambda_{i}}}\left|r_{i}\right|
\end{aligned}
$$

where $\widetilde{e}_{j}(t)=\left[\widetilde{e}_{j 1}, \widetilde{e}_{j 2}, \ldots, \widetilde{e}_{j N}\right]^{T}$ be the column vector of the $i$ th $e(t)$.

Using $\operatorname{sign}\left(e_{i}\right)=\left(\operatorname{sign}\left(e_{i 1}\right), \operatorname{sign}\left(e_{i 2}\right), \cdots, \operatorname{sign}\left(e_{i n}\right)\right)^{T}$ and Lemma 2 , we have

$$
\dot{V}(t) \leq \sum_{j=1}^{n} p_{j} \widetilde{e}_{j}^{T} Z_{j} \widetilde{e}_{j}^{T}-\bar{k} \sum_{i=1}^{N} \sqrt{\lambda \max (P)}\left\|e_{i}\right\|-\sum_{i=1}^{N} \frac{\bar{k}}{\sqrt{\lambda_{i}}}\left|r_{i}\right|,
$$

$Z_{j}$ is defined as

$$
Z_{j}=\theta_{j} I_{N}-\Xi+c \gamma_{j} A, \quad j=1,2, \ldots, n
$$

Using Lemma 2 and Lemma 4, we have

$$
\dot{V}(t) \leq \sum_{j=1}^{n} p_{j} \widetilde{e}_{j}^{T} Z_{j} \widetilde{e}_{j}^{T}-\sqrt{2} \bar{k}\left(\frac{1}{2} \sum_{i=1}^{N} e_{i}^{T} P e_{i}+\frac{1}{2} \sum_{i=1}^{N} \frac{1}{\lambda_{i}} r_{i}^{2}\right)^{\frac{1}{2}} .
$$

It follows from condition (3.5) that

$$
Z_{j} \leq 0
$$

which shows that $\dot{V}(t) \leq-\sqrt{2} \bar{k} V^{\frac{1}{2}}(t)$.

When $(l+\theta) T \leq t<(l+1) T$, for $l \in \ell$, we have

$$
\begin{aligned}
\dot{V}(t) & =\sum_{i=1}^{N} e_{i}^{T} P \dot{e}_{i} \\
& =\sum_{i=1}^{N}\left\{e_{i}^{T} P\left[f\left(y_{i}\right)-f\left(x_{i}\right)-\Theta e_{i}\right]+e_{i}^{T} P \Theta e_{i}+c \sum_{j=1}^{N} a_{i j} e_{i}^{T} P \Gamma e_{j}\right\} \\
& \leq-\xi \sum_{i=1}^{N} e_{i}^{T} e_{i}+\sum_{j=1}^{n} p_{j} \widetilde{e}_{j}^{T} \theta_{j} I_{N} \widetilde{e}_{j}+c \sum_{j=1}^{n} p_{j} \widetilde{e}_{j}^{T} \gamma_{j} A \widetilde{e}_{j} \\
& \leq-\sum_{j=1}^{n} p_{j} \widetilde{e}_{j}^{T} \frac{\xi}{\lambda \max (P)} I_{N} \widetilde{e}_{j}+\sum_{j=1}^{n} p_{j} \widetilde{e}_{j}^{T} \theta_{j} I_{N} \widetilde{e}_{j}+c \sum_{j=1}^{n} p_{j} \widetilde{e}_{j}^{T} \gamma_{j} A \widetilde{e}_{j} \\
& =\sum_{j=1}^{n} p_{j} \widetilde{e}_{j}^{T} S_{j} \widetilde{e}_{j},
\end{aligned}
$$

where $\widetilde{e}_{j}(t)=\left[\widetilde{e}_{j 1}, \widetilde{e}_{j 2}, \ldots, \widetilde{e}_{j N}\right]^{T}$ be the column vector of the $i$ th $e(t), S_{j}$ is defined as

$$
S_{j}=\theta_{j} I_{N}-\frac{\xi}{\lambda_{\max }(P)} I_{N}+c \gamma_{j} A, \quad j=1,2, \ldots, n .
$$


It follows from condition (3.6) that

$$
S_{j} \leq 0
$$

which shows that $\dot{V}(t) \leq 0$.

Namely, let $\alpha=\sqrt{2} \bar{k}, 1-\eta=\frac{1}{2}$, we have

$$
\begin{cases}\dot{V}(t) \leq-\alpha V^{\eta}(t), & l T \leq t \leq l T+\theta T \\ \dot{V}(t) \leq 0, & l T+\theta T \leq t<(l+1) T .\end{cases}
$$

This implies the conclusion by Lemma 3 and the proof is complete.

Remark 1. Obviously, when $\theta=1$, the intermittent control (3.2) is degenerated to a continuous control input which has been extensively proposed in previous work (see [15, 41-48]). And in this case, it is clear from Theorem 1 that system (2.1) and system (3.1) are finite-time synchronized under Assumption 1 based on the continuous control and the main results in Refs. [41-48] can be easily analogized from the simplified criteria despite model differences.

Remark 2. In this paper, we introduce finite-time intermittent control technique to synchronize complex networks. In the literature, there are many results concerning exponential synchronization via intermittent control (see [29-32, 39-40]). However, to the best of our knowledge, there is few published paper dealing with finite-time synchronization between two complex networks via intermittent control. Obviously, our results can enrich the finitetime synchronization problem of network by the intermittent control. What's more, compare with the exponential synchronization between two complex networks via intermittent control, the setting time of synchronization by finite-time intermittent control is estimated in finite-time.

Remark 3. From (3.7), we can see the roles that the control rate $\theta$ and the tunable constant $\bar{k}$ are played an important role in finite-time synchronization of complex networks. The equation (3.7) indicates that the convergence time is changing when the values of $\bar{k}$ and $\theta$ increases. Denote $T(\theta)=\frac{2 V^{\frac{1}{2}}(0)}{\sqrt{2} \bar{k} \theta}$, if we fixed the $\bar{k}$, then we have, $T^{\prime}(\theta)=-\frac{2 V^{\frac{1}{2}}(0)}{\sqrt{2} \bar{k} \theta^{2}}<0$. Obviously, $T(\theta)(0<\theta<1)$ is a strictly monotone decreasing function. Hence, the larger the control rate is, the shorter in the convergence time to achieve finite-time synchronization of complex networks will be. In addition, the synchronization for the complex dynamical networks with a continuous control input (where $\theta=1$ ) is faster in the convergence time than those of complex networks with periodically intermittent control $(0<\theta<1)$.

Remark 4. In Ref. [15], U.E. Vincent et.al. studied the finite-time synchronization for a class of chaotic and hyperchaotic systems via adaptive feedback controller. To derive their results, $l=\frac{\lambda}{c}>0$ is selected such that $V^{\eta}\left(w, k_{1}\right) \leq l V_{1}^{\eta}(w)$ in Ref. [15]. Evidently, the application scope and generalization domain of the results are limited due to a suitable 
parameter should be found. Especially, the system is not a chaotic system, but a class of complex dynamical networks or neural networks, the method of Ref. [15] is not suitable. However, our results do not need this restriction by introducing a new controller and a novel updated law.

In Ref. [34], Zhu et al. investigated the stabilization and synchronization of chaotic systems via intermittent control. He only concerned asymptotically stable under adaptive intermittent controlling. In Theorem 1, we introduce a novel periodically intermittent adaptive controlling to guarantee finite-time synchronization.

Remark 5. From the inequality (3.5), we have $\lambda_{\max }(\Xi) \geq c \lambda_{\max }\left(\gamma_{j} A\right)+\max _{1 \leq j \leq n} \theta_{j}$, from the inequality (3.6), we have $\frac{\xi}{\lambda_{\max (P)}} \geq c \lambda_{\max }\left(\gamma_{j} A\right)+\max _{1 \leq j \leq n} \theta_{j}$. Since $\lambda_{\max }(\Xi)$ and $\frac{\xi}{\lambda_{\max }(P)}$ are two different values, then the two conditions are needed in Theorem 1 . But, if the two different values satisfy dependent relation, (3.5) and (3.6) are no difference in some sense. If $\lambda_{\max }(\Xi) \geq \frac{\xi}{\lambda_{\max }(P)}$, then we have the following Corollary 1. If $\frac{\xi}{\lambda_{\max }(P)} \geq \lambda_{\max }(\Xi)$, then we have the following Corollary 2 .

Corollary 1. Suppose Assumption 1 holds. If there exist a positive definite diagonal matrix $P>0$ and $\lambda_{\max }(\Xi) \geq \frac{\xi}{\lambda_{\max }(P)}\left(\Xi=\operatorname{diag}\left(\eta_{1}, \eta_{2}, \ldots, \eta_{N}\right)>0\right)$ such that the following conditions hold:

$$
\theta_{j} I_{N}-\frac{\xi}{\lambda_{\max }(P)} I_{N}+c \gamma_{j} A \leq 0, \quad j=1,2, \ldots, n,
$$

where $\Gamma=\operatorname{diag}\left(\gamma_{1}, \gamma_{2}, \ldots, \gamma_{n}\right), \Theta=\operatorname{diag}\left(\theta_{1}, \theta_{2}, \ldots, \theta_{n}\right), \xi$ is a positive constant satisfies Assumption 1, and $I_{N}$ is an appropriate identity matrix. Then the error system (3.3) is synchronized under the periodically intermittent controller (3.2) and the intermittent updated law (3.4) in a finite time:

$$
T_{1}=\frac{2 V^{1 / 2}(0)}{\sqrt{2 \bar{k}} \theta},
$$

where $V(0)=\frac{1}{2} \sum_{i=1}^{N} e_{i}^{T}(0) P e_{i}(0)+\frac{1}{2} \sum_{i=1}^{N} \frac{1}{\lambda_{i}}\left(r_{i}(0)\right)^{2}, e_{i}(0), r_{i}(0)$ are the initial conditions of $e_{i}, r_{i}$.

Corollary 2. Suppose Assumption 1 holds. If there exist a positive definite diagonal matrix $P>0$ and $\frac{\xi}{\lambda_{\max }(P)} \geq \lambda_{\max }(\Xi)\left(\Xi=\operatorname{diag}\left(\eta_{1}, \eta_{2}, \ldots, \eta_{N}\right)>0\right)$ such that the following conditions hold:

$$
\theta_{j} I_{N}-\Xi+c \gamma_{j} A \leq 0, \quad j=1,2, \ldots, n,
$$

where $\Gamma=\operatorname{diag}\left(\gamma_{1}, \gamma_{2}, \ldots, \gamma_{n}\right), \Theta=\operatorname{diag}\left(\theta_{1}, \theta_{2}, \ldots, \theta_{n}\right), \xi$ is a positive constant satisfies Assumption 1, and $I_{N}$ is an appropriate identity matrix. Then the error system (3.3) is synchronized under the periodically intermittent controller (3.2) and the intermittent updated 
law (3.4) in a finite time:

$$
T_{1}=\frac{2 V^{1 / 2}(0)}{\sqrt{2} \bar{k} \theta},
$$

where $V(0)=\frac{1}{2} \sum_{i=1}^{N} e_{i}^{T}(0) P e_{i}(0)+\frac{1}{2} \sum_{i=1}^{N} \frac{1}{\lambda_{i}}\left(r_{i}(0)\right)^{2}, e_{i}(0), r_{i}(0)$ are the initial conditions of $e_{i}, r_{i}$.

If we let the adaptive update laws $r_{i}=0(i=1,2, \ldots, N)$, for all $t \geq 0$, then we have the following Corollary 3.

Corollary 3. Suppose Assumption 1 holds. If there exist a positive definite matrix $\Xi=\operatorname{diag}\left(\eta_{1}, \eta_{2}, \ldots, \eta_{N}\right)>0$ and a positive definite diagonal matrix $P>0$ such that the following conditions hold:

$$
\begin{gathered}
\theta_{j} I_{N}-\Xi+c \gamma_{j} A<0, \quad j=1,2, \ldots, n, \\
\theta_{j} I_{N}-\frac{\xi}{\lambda_{\max }(P)} I_{N}+c \gamma_{j} A<0, \quad j=1,2, \ldots, n,
\end{gathered}
$$

where $\Gamma=\operatorname{diag}\left(\gamma_{1}, \gamma_{2}, \ldots, \gamma_{n}\right), \Theta=\operatorname{diag}\left(\theta_{1}, \theta_{2}, \ldots, \theta_{n}\right), \xi$ is a positive constant satisfies Assumption 1, and $I_{N}$ is an appropriate identity matrix. Then the error system (3.3) is synchronized under the periodically intermittent controller

$$
\left\{\begin{array}{l}
u_{i}(t)=-\eta_{i} e_{i}-\bar{k} \frac{\sqrt{\lambda_{\max }(P)}}{{ }^{\lambda} \min ^{(P)}} \operatorname{sign}\left(e_{i}\right), l T \leq t<l T+\delta, \\
u_{i}(t)=0, \quad\left\|e_{i}\right\|=0 \text { or } l T+\delta \leq t<(l+1) T
\end{array}\right.
$$

in a finite time:

$$
T_{2}=\frac{2 V^{1 / 2}(0)}{\sqrt{2} \bar{k} \theta}
$$

where $V(0)=\frac{1}{2} \sum_{i=1}^{N} e_{i}^{T}(0) P e_{i}(0), e_{i}(0)$ is the initial condition of $e_{i}(t)$.

The proof of this corollary is easily obtained from Theorem 1 . Thus it is omitted here.

\section{Finite-time synchronization of complex networks via intermittent feedback control}

In this section, we shall focus on analyzing finite-time synchronization between two complex dynamical networks via periodically intermittent feedback control, and found an adaptive law to automatically force the error systems converge to zero within a finite time and remain on it forever.

Now, we consider the following two complex dynamical networks:

$$
\begin{cases}\dot{x}_{i}=f\left(x_{i}\right)+c \sum_{j=1}^{N} a_{i j} \Gamma x_{j}-r_{i} e_{i}, & i=1,2, \cdots, N, \\ \dot{y}_{i}=f\left(y_{i}\right)+c \sum_{j=1}^{N} a_{i j} \Gamma y_{j}+k_{i} e_{i}, & i=1,2, \cdots, N,\end{cases}
$$


where $r_{i}, k_{i}$ are the intermittent feedback gain which can be derived as:

$$
\begin{aligned}
& \begin{cases}\dot{k}_{i}=-\psi_{i}\left[e_{i}^{T} P e_{i}+\frac{\bar{k} k_{i} \sqrt{\lambda_{\max }(P)} e_{i}^{T}}{k_{i}^{2}} \operatorname{sign}\left(e_{i}\right)+\frac{\bar{k}}{\sqrt{\psi_{i}}} \operatorname{sign}\left(k_{i}\right)\right], & l T \leq t<l T+\delta, \\
\dot{k}_{i}=0, & l T+\delta \leq t<(l+1) T,\end{cases} \\
& \begin{cases}\dot{r}_{i}=-\varphi_{i}\left[e_{i}^{T} P e_{i}+\frac{r_{i} \eta_{i} e_{i}^{T} P e_{i}}{r_{i}^{2}}+\frac{\bar{k}}{\sqrt{\varphi_{i}}} \operatorname{sign}\left(r_{i}\right)\right], & l T \leq t<l T+\delta, \\
\dot{r}_{i}=0, & l T+\delta \leq t<(l+1) T,\end{cases}
\end{aligned}
$$

for $\psi_{i}, \varphi_{i}>0(i=1,2, \ldots, N)$ are arbitrary constants, $\bar{k}>0$ is a tunable constant, $\Xi=\operatorname{diag}\left(\eta_{1}, \eta_{2}, \ldots, \eta_{N}\right)>0$ is a positive definite matrix.

Then we can derive the following result.

Theorem 2. Suppose Assumption 1 holds. If there exist a positive definite matrix $\Xi=$ $\operatorname{diag}\left(\eta_{1}, \eta_{2}, \ldots, \eta_{N}\right)>0$ and a positive definite diagonal matrix $P>0$ such that the following conditions hold:

$$
\begin{gathered}
\theta_{j} I_{N}-\Xi+c \gamma_{j} A \leq 0, \quad j=1,2, \ldots, n, \\
\theta_{j} I_{N}-\frac{\xi}{\lambda_{\max }(P)} I_{N}+c \gamma_{j} A \leq 0, \quad j=1,2, \ldots, n,
\end{gathered}
$$

where $\Gamma=\operatorname{diag}\left(\gamma_{1}, \gamma_{2}, \ldots, \gamma_{n}\right), \Theta=\operatorname{diag}\left(\theta_{1}, \theta_{2}, \ldots, \theta_{n}\right), \xi$ is a positive constant satisfies Assumption 1 , and $I_{N}$ is an appropriate identity matrix. Then under the periodically intermittent feedback gain control (4.2) and (4.3), the two complex networks are synchronized in a finite time:

$$
T_{3}=\frac{2 V^{1 / 2}(0)}{\sqrt{2} \bar{k} \theta}
$$

where $V(0)=\frac{1}{2} \sum_{i=1}^{N} e_{i}^{T}(0) P e_{i}(0)+\frac{1}{2} \sum_{i=1}^{N} \frac{1}{\psi_{i}}\left(k_{i}(0)\right)^{2}+\frac{1}{2} \sum_{i=1}^{N} \frac{1}{\varphi_{i}}\left(r_{i}(0)\right)^{2}, e_{i}(0), k_{i}(0), r_{i}(0)$ are the initial conditions of $e_{i}, k_{i}, r_{i}$.

Proof. According to the the systems (4.1), then error dynamical system can be derived as

$$
\left\{\begin{array}{l}
\dot{e}_{i}=f\left(y_{i}\right)-f\left(x_{i}\right)+c \sum_{j=1}^{N} a_{i j} \Gamma e_{j}(t)+k_{i} e_{i}+r_{i} e_{i} \\
l T \leq t<l T+\theta T, \quad i=1,2, \cdots, N \\
\dot{e}_{i}=f\left(y_{i}\right)-f\left(x_{i}\right)+c \sum_{j=1}^{N} a_{i j} \Gamma e_{j} \\
l T+\theta T \leq t<(l+1) T, \quad i=1,2, \cdots, N
\end{array}\right.
$$

We choose a positive definite function in the form of

$$
V(t)=\frac{1}{2} \sum_{i=1}^{N} e_{i}^{T} P e_{i}+\frac{1}{2} \sum_{i=1}^{N} \frac{1}{\psi_{i}} k_{i}^{2}+\frac{1}{2} \sum_{i=1}^{N} \frac{1}{\varphi_{i}} r_{i}^{2} .
$$


Then the derivative of $V(t)$ with respect to time $t$ along the solutions of Eq. (4.7) can be calculated as follows:

When $l T \leq t<(l+\theta) T$, for $l \in \ell$,

$$
\begin{aligned}
\dot{V}(t)= & \sum_{i=1}^{N} e_{i}^{T} P e_{i}+\sum_{i=1}^{N} \frac{1}{\psi_{i}} k_{i} \dot{k}_{i}+\sum_{i=1}^{N} \frac{1}{\varphi_{i}} r_{i} \dot{r}_{i} \\
= & \sum_{i=1}^{N} e_{i}^{T} P\left[f\left(y_{i}\right)-f\left(x_{i}\right)+c \sum_{j=1}^{N} a_{i j} \Gamma e_{j}+k_{i} e_{i}+r_{i} e_{i}\right]-\sum_{i=1}^{N} k_{i}\left[e_{i}^{T} P e_{i}+\right. \\
& \left.\frac{\bar{k}}{\sqrt{\psi_{i}}} \operatorname{sign}\left(k_{i}\right)+\frac{\bar{k} k_{i} \sqrt{\lambda \max (P)} e_{i}^{T}}{k_{i}^{2}} \operatorname{sign}\left(e_{i}\right)\right]-\sum_{i=1}^{N} r_{i}\left[e_{i}^{T} P e_{i}+\frac{r_{i} \eta_{i} e_{i}^{T} P e_{i}}{r_{i}^{2}}+\right. \\
& \left.\frac{\bar{k}}{\sqrt{\varphi_{i}}} \operatorname{sign}\left(r_{i}\right)\right] \\
= & \sum_{i=1}^{N} e_{i}^{T} P\left[f\left(y_{i}\right)-f\left(x_{i}\right)+c \sum_{j=1}^{N} a_{i j} \Gamma e_{j}\right]-\sum_{i=1}^{N} \bar{k} \sqrt{\lambda_{\max }(P)} e_{i}^{T} \operatorname{sign}\left(e_{i}\right)- \\
& \sum_{i=1}^{N} \frac{\bar{k}}{\sqrt{\psi_{i}}} k_{i} \operatorname{sign}\left(k_{i}\right)-\sum_{i=1}^{N} e_{i}^{T} \eta_{i} P e_{i}-\sum_{i=1}^{N} \frac{\bar{k}}{\sqrt{\varphi_{i}}} r_{i} \operatorname{sign}\left(r_{i}\right) \\
= & \sum_{i=1}^{N}\left\{e_{i}^{T} P\left[f\left(y_{i}\right)-f\left(x_{i}\right)-\Theta e_{i}\right]+e_{i}^{T} P \Theta e_{i}+c \sum_{j=1}^{N} a_{i j} \Gamma e_{j}\right\}-\sum_{i=1}^{N} \frac{\bar{k}}{\sqrt{\psi_{i}}} k_{i} \operatorname{sign}\left(k_{i}\right) \\
& -\sum_{i=1}^{N} \bar{k} \sqrt{\lambda \max (P)} e_{i}^{T} \operatorname{sign}\left(e_{i}\right)-\sum_{i=1}^{N} e_{i}^{T} \eta_{i} P e_{i}-\sum_{i=1}^{N} \frac{\bar{k}}{\sqrt{\varphi_{i}}} r_{i} \operatorname{sign}\left(r_{i}\right) \\
\leq & -\xi \sum_{i=1}^{N} e_{i}^{T} e_{i}+\sum_{i=1}^{N} e_{i}^{T}\left(P \Theta-\eta_{i} P\right) e_{i}+c \sum_{i=1}^{N} e_{i}^{T} P \sum_{j=1}^{N} a_{i j} \Gamma e_{j}-\sum_{i=1}^{N} \frac{\bar{k}}{\sqrt{\psi_{i}}} k_{i} \operatorname{sign}\left(k_{i}\right) \\
& -\sum_{i=1}^{N} \bar{k} \sqrt{\lambda \max (P)} e_{i}^{T} \operatorname{sign}\left(e_{i}\right)-\sum_{i=1}^{N} \frac{\bar{k}}{\sqrt{\varphi_{i}}} r_{i} \operatorname{sign}\left(r_{i}\right), \\
\sim &
\end{aligned}
$$

where $\widetilde{e}_{j}=\left[\widetilde{e}_{j 1}, \widetilde{e}_{j 2}, \ldots, \widetilde{e}_{j N}\right]^{T}$ be the column vector of the $i$ th $e(t)$.

Using $\operatorname{sign}\left(e_{i}\right)=\left(\operatorname{sign}\left(e_{i 1}\right), \operatorname{sign}\left(e_{i 2}\right), \cdots, \operatorname{sign}\left(e_{i n}\right)\right)^{T}$ and Lemma 2 , we have

$$
\dot{V}(t) \leq \sum_{j=1}^{n} p_{j} \widetilde{e}_{j}^{T} Z_{j} \widetilde{e}_{j}^{T}-\bar{k} \sum_{i=1}^{N} \sqrt{\lambda_{\max }(P)}\left\|e_{i}\right\|-\sum_{i=1}^{N} \frac{\bar{k}}{\sqrt{\psi_{i}}}\left|k_{i}\right|-\sum_{i=1}^{N} \frac{\bar{k}}{\sqrt{\varphi_{i}}}\left|r_{i}\right|,
$$

$Z_{j}$ is defined as

$$
Z_{j}=\theta_{j} I_{N}-\Xi+c \gamma_{j} A, \quad j=1,2, \ldots, n .
$$

Using Lemma 2 and Lemma 4, we have

$$
\dot{V}(t) \leq \sum_{j=1}^{n} p_{j} \widetilde{e}_{j}^{T} Z_{j} \widetilde{e}_{j}^{T}-\sqrt{2} \bar{k}\left(\frac{1}{2} \sum_{i=1}^{N} e_{i}^{T} P e_{i}+\frac{1}{2} \sum_{i=1}^{N} \frac{1}{\psi_{i}} k_{i}^{2}+\frac{1}{2} \sum_{i=1}^{N} \frac{1}{\varphi_{i}} r_{i}^{2}\right)^{\frac{1}{2}} .
$$


It follows from condition (4.4) that

$$
Z_{j} \leq 0
$$

which shows that $\dot{V}(t) \leq-\sqrt{2} \bar{k} V^{\frac{1}{2}}(t)$.

When $(l+\theta) T \leq t<(l+1) T$, for $l \in \ell$, we have

$$
\begin{aligned}
\dot{V}(t) & =\sum_{i=1}^{N} e_{i}^{T} P \dot{e}_{i} \\
& =\sum_{i=1}^{N}\left\{e_{i}^{T} P\left[f\left(y_{i}\right)-f\left(x_{i}\right)-\Theta e_{i}\right]+e_{i}^{T} P \Theta e_{i}+c \sum_{j=1}^{N} a_{i j} e_{i}^{T} P \Gamma e_{j}\right\} \\
& \leq-\xi \sum_{i=1}^{N} e_{i}^{T} e_{i}+\sum_{j=1}^{n} p_{j} \widetilde{e}_{j}^{T} \theta_{j} I_{N} \widetilde{e}_{j}+c \sum_{j=1}^{n} p_{j} \widetilde{e}_{j}^{T} \gamma_{j} A \widetilde{e}_{j} \\
& \leq-\sum_{j=1}^{n} p_{j} \widetilde{e}_{j}^{T} \frac{\xi}{\lambda \max (P)} I_{N} \widetilde{e}_{j}+\sum_{j=1}^{n} p_{j} \widetilde{e}_{j}^{T} \theta_{j} I_{N} \widetilde{e}_{j}+c \sum_{j=1}^{n} p_{j} \widetilde{e}_{j}^{T} \gamma_{j} A \widetilde{e}_{j} \\
& =\sum_{j=1}^{n} p_{j} \widetilde{e}_{j}^{T} S_{j} \widetilde{e}_{j},
\end{aligned}
$$

where $\widetilde{e}_{j}=\left[\widetilde{e}_{j 1}, \widetilde{e}_{j 2}, \ldots, \widetilde{e}_{j N}\right]^{T}$ be the column vector of the $i$ th $e(t), S_{j}$ is defined as

$$
S_{j}=\theta_{j} I_{N}-\frac{\xi}{\lambda \max (P)} I_{N}+c \gamma_{j} A, \quad j=1,2, \ldots, n .
$$

It follows from condition (4.5) that

$$
S_{j} \leq 0
$$

which shows that $\dot{V}(t) \leq 0$.

Namely, let $\alpha=\sqrt{2} \bar{k}, 1-\eta=\frac{1}{2}$, we have

$$
\begin{cases}\dot{V}(t) \leq-\alpha V^{\eta}(t), & l T \leq t \leq l T+\theta T \\ \dot{V}(t) \leq 0, & l T+\theta T \leq t<(l+1) T .\end{cases}
$$

According to Lemma 4, Then this completes the proof.

Remark 6. The intermittent feedback gains $k_{i}$ and $r_{i}$ are automatically adapted to the suitable strength $k_{0}$ and $r_{0}$, respectively, which depend on the initial values. In Ref. [17], $\mathrm{Xu}$ et. al. considered the two complex networks by the adjustment of feedback gains $k_{i}$ and $r_{i}$ such that the error system (8) asymptotically converge to zero. However, in Theorem 2 of this paper, we can consider the same model by using the adapted of feedback gains $k_{i}$ and $r_{i}$ drive the error system (4.7) in finite time converge to zero. What's more, the Theorem 2 of this paper is suitable to a great many complex dynamical networks, neural networks and chaotic systems concerning about intermittent control. For example, the method of Theorem 
2 in this paper is suitable to the main results of Theorems and corollaries in Ref. [46].

Remark 7. According to the convergence time $T_{1}, T_{2}, T_{3}$, we can conclude the convergence time satisfies: $T_{2} \leq T_{1} \leq T_{3}$. In Theorem 1, we did shorten the synchronization or convergence time by using the intermittent adaptive controller in contrast to the Theorem 2, which introduced the intermittent feedback controller, but using many control laws is very expensive in practical applications. Besides, a very interesting phenomenon is revealed that the more the control gains are, the longer in time to achieve finite-time synchronization will be.

\section{Numerical examples}

In the previous section, theorems essentially provide the criteria for finite-time synchronization. In this section, we use representative example to illustrate how these theorem can be applied to achieve finite-time synchronization in complex network.

We consider two complex networks, in which each subsystem is a Lorenz system. The dynamics of a single Lorenz system is described as

$$
\left[\begin{array}{l}
\dot{x}_{1} \\
\dot{x}_{2} \\
\dot{x}_{3}
\end{array}\right]=\left[\begin{array}{lll}
-r_{1} & r_{1} & 0 \\
r_{3} & -1 & 0 \\
0 & 0 & -r_{2}
\end{array}\right]\left[\begin{array}{l}
x_{1} \\
x_{2} \\
x_{3}
\end{array}\right]+\left[\begin{array}{l}
0 \\
-x_{1} x_{3} \\
x_{1} x_{2}
\end{array}\right]=f(x),
$$

where the parameters are selected as $r_{1}=10, r_{3}=28, r_{2}=8 / 3$, the single Lorenz system has a chaotic attractor (Fig. 2). We take $P=\operatorname{diag}(5,4,2)$ and $\Theta=\operatorname{diag}(3,3,3)$ such that the Lorenz system satisfies Assumption 1.

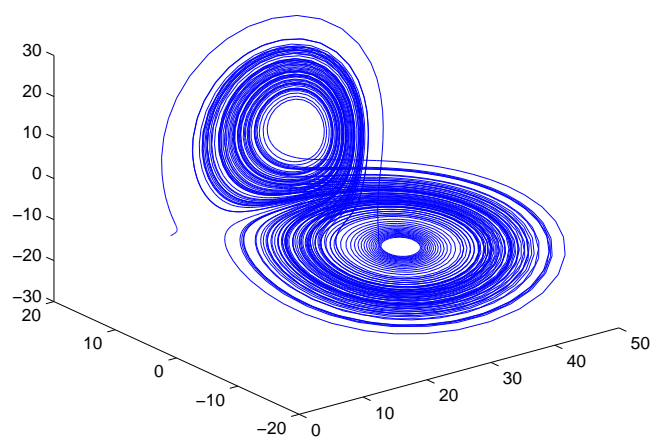

Fig.2. The chaotic trajectories of the Lorenz system.

\subsection{The simulation with the periodically intermittent adaptive controllers}

Example 1. We consider the directed network with 100 dynamical nodes. $A=\left(a_{i j}\right)_{100 \times 100}$ 
is a symmetrically diffusive coupling matrix with $a_{i j}=0$ or $1(j \neq i), \Gamma=\operatorname{diag}\{1,1,1\}$ and the coupling strength $c=1$.

According to Theorem 1 in Section 3, one can synchronize the error system (3.3) by design of the following intermittent adaptive controllers and intermittent updated laws

$$
\left\{\begin{array}{l}
u_{i}(t)=r_{i} e_{i}-\bar{k} \frac{\sqrt{\lambda \max (P)}}{{ }^{\lambda} \min ^{(P)}} \operatorname{sign}\left(e_{i}\right), l T \leq t<l T+\delta \\
u_{i}(t)=0, \quad\left\|e_{i}\right\|=0 \text { or } l T+\delta \leq t<(l+1) T
\end{array}\right.
$$

and

$$
\left\{\begin{array}{l}
\dot{r}_{i}=-\lambda_{i}\left[\frac{\eta_{i} r_{i} e_{i}^{T} P e_{i}}{r_{i}^{2}}+e_{i}^{T} P e_{i}+\frac{\bar{k}}{\sqrt{\lambda_{i}}} \operatorname{sign}\left(r_{i}\right)\right], l T \leq t<l T+\delta, \\
\dot{r}_{i}=0, \quad\left\|r_{i}\right\|=0 \text { or } l T+\delta \leq t<(l+1) T
\end{array}\right.
$$

In the simulation, all parameters are selected as: $\bar{k}=10, \lambda_{i}=1, r_{i}(0)=2+0.5 i, x_{i}(0)=$ $(3+0.2 i, 4+0.3 i, 6+0.4 i)^{T}, y_{i}(0)=(-5+0.7 i,-1+0.8 i,-4+0.9 i)^{T}$, where $1 \leq i \leq 100$. Further, select $T=0.2$ and $\delta=0.16$. Under above conditions, all assumptions of Theorem 1 are satisfied, then system (2.1) and system (3.1) are finite-time synchronized under the intermittent adaptive controllers (5.2) and the intermittent updated laws (5.3), which is shown in Fig 3.
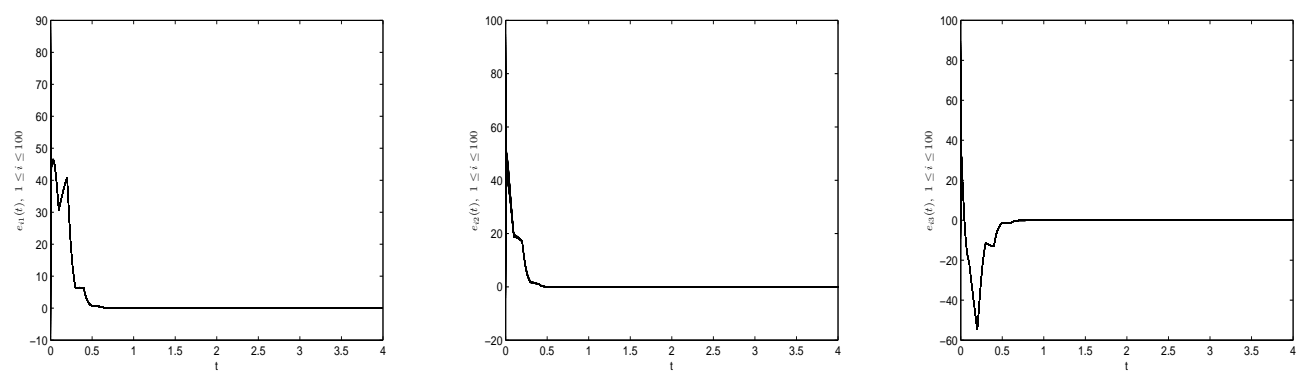

Fig.3. The errors $e_{i 1}, e_{i 2}, e_{i 3}, i=1,2, \ldots, 100$ under periodically intermittent adaptive controllers (5.2) and (5.3) with the parameters $T=0.2, \delta=0.16$.
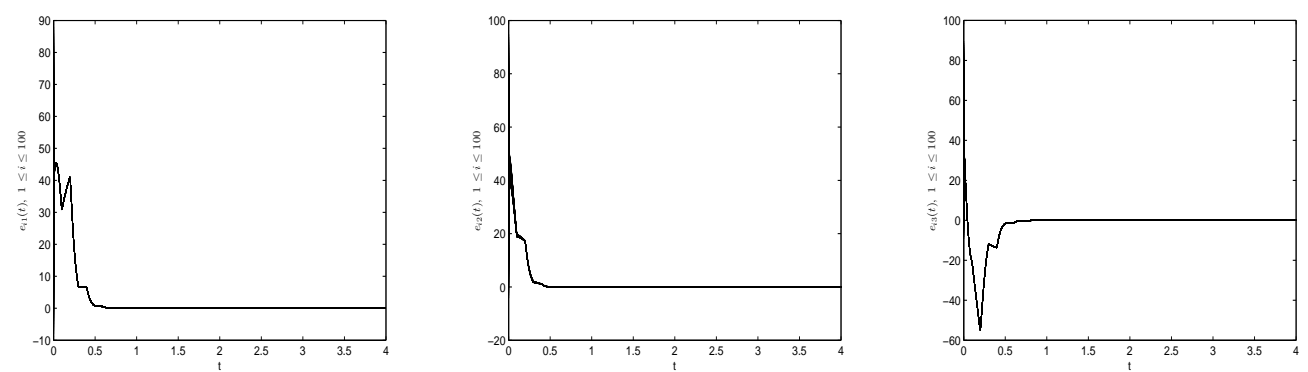

Fig.4. The errors $e_{i 1}, e_{i 2}, e_{i 3}, i=1,2, \ldots, 100$ under periodically intermittent adaptive controllers (5.2) and (5.3) with the parameters $T=0.2, \delta=0.08$. 


\subsection{The simulation with the periodically intermittent feedback controllers}

Example 2. Consider the following example consisting of 100 identical nodes, which is described by:

$$
\begin{aligned}
& \left\{\begin{array}{l}
\dot{y}_{i 1}=r_{1}\left(y_{i 2}-y_{i 1}\right)+r_{3} \sum_{j=1}^{100} a_{i j} \Gamma y_{i j}+k_{i} e_{i 1}, \\
\dot{y}_{i 2}=r_{3} y_{i 1}-y_{i 2}-y_{i 1} y_{i 3}+c \sum_{j=1}^{100} a_{i j} \Gamma y_{i j}+k_{i} e_{i 2}, \\
\dot{y}_{i 3}=-r_{2} y_{i 3}+y_{i 1} y_{i 2}+c \sum_{j=1}^{100} a_{i j} \Gamma y_{i j}+k_{i} e_{i 3},
\end{array}\right. \\
& \left\{\begin{array}{l}
\dot{x}_{i 1}=r_{1}\left(x_{i 2}-x_{i 1}\right)+c \sum_{j=1}^{100} a_{i j} \Gamma x_{i j}-r_{i} e_{i 1}, \\
\dot{x}_{i 2}=r_{3} x_{i 1}-x_{i 2}-x_{i 1} x_{i 3}+c \sum_{j=1}^{100} a_{i j} \Gamma x_{i j}-r_{i} e_{i 2}, \\
\dot{x}_{i 3}=-r_{2} x_{i 3}+x_{i 1} x_{i 2}+c \sum_{j=1}^{100} a_{i j} \Gamma x_{i j}-r_{i} e_{i 3},
\end{array}\right.
\end{aligned}
$$

where $i=1,2, \ldots, 100, A=\left(a_{i j}\right)_{100 \times 100}$ is a symmetrically diffusive coupling matrix with $a_{i j}=0$ or $1(j \neq i), \Gamma=\operatorname{diag}\{1,1,1\}$ and the coupling strength $c=1$.

According to Theorem 2, the intermittent updated laws of the coupling strengths can be described by (4.2)-(4.3).

For convenience, in the numerical simulation, all parameters are given as follows: $k_{i}(0)=$ $-2+3 i, r_{i}(0)=-1+2 i, \psi_{i}=1, \varphi_{i}=1, x_{i}(0)=(4+0.2 i, 2+0.3 i, 5+0.4 i)^{T}, y_{i}(0)=$ $(-4+0.7 i,-3+0.8 i,-5+0.9 i)^{T}, T=0.2, \delta=0.16$, where $1 \leq i \leq 10$. The discontinuous sign function is replaced by a continuous function (tanh). Then system (5.4) and (5.5) are finite-time synchronized under the periodically intermittent feedback controllers (4.2) and (4.3), which is shown in Fig. 5.
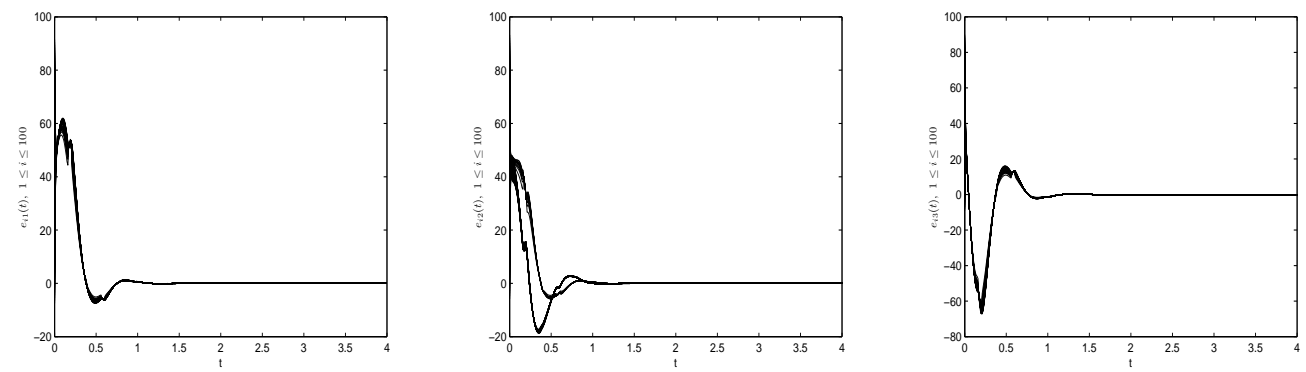

Fig.5. The synchronization errors $e_{i 1}, e_{i 2}, e_{i 3}, i=1,2, \ldots, 100$ of the complex networks (5.4) and (5.5) with $T=0.2, \delta=0.16$.
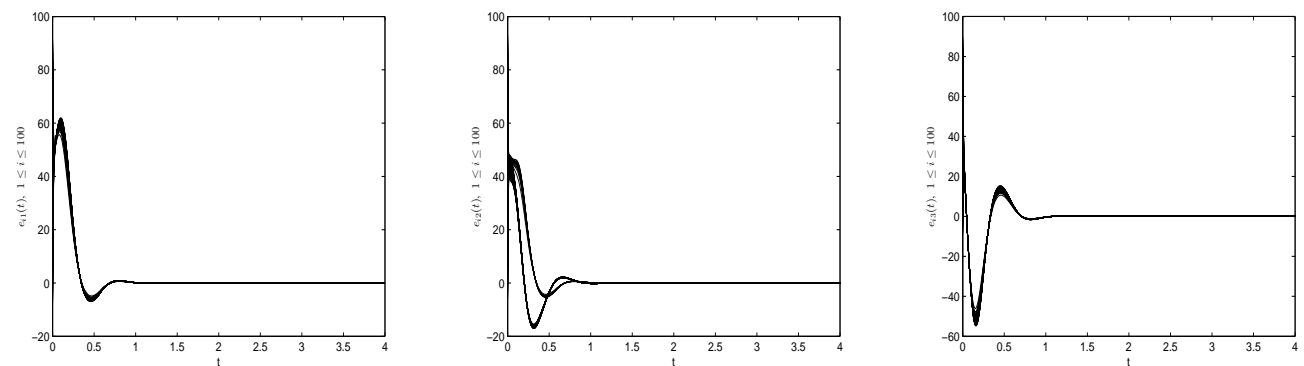
Fig.6. The synchronization errors $e_{i 1}, e_{i 2}, e_{i 3}, i=1,2, \ldots, 100$ of the complex networks (5.4) and (5.5) with full control (4.2) and (4.3).
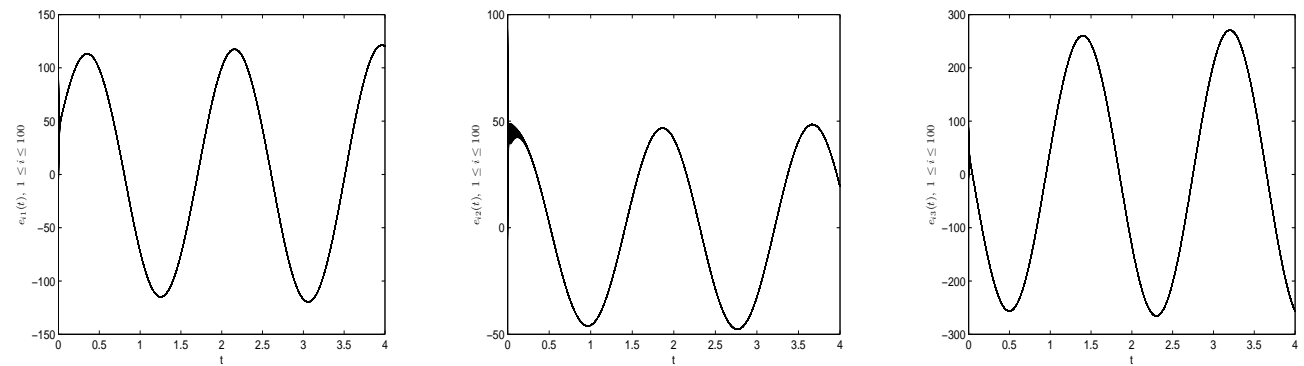

Fig.7. The synchronization errors $e_{i 1}, e_{i 2}, e_{i 3}, i=1,2, \ldots, 100$ of the complex networks (5.4) and (5.5) with no control inputs.
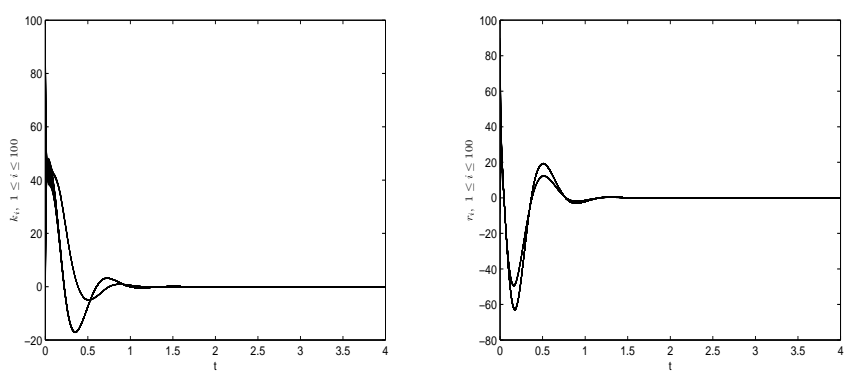

Fig.8. The feedback gains $k_{i}, r_{i}, i=1,2, \ldots, 100$ of the complex networks (4.2) and (4.3) with

$$
T=0.2, \delta=0.16 \text {. }
$$

Fig.5 shows the synchronous errors $e_{i j}(1 \leq j \leq 3)$ with intermittent control under $T=$ $0.2, \delta=0.16$. Fig.6 shows the synchronous errors $e_{i j}(1 \leq j \leq 3)$ with full control and the no control inputs are revealed in Fig.7, the feedback gains $k_{i}, r_{i}$ are shown in Fig.8. It is clear that the synchronization of full control is faster than intermittent control.

Remark 8. Through simple computation by the above parameters, we get the setting time $\left(T_{1}, T_{2}, T_{3}\right)=(0.875,0.786,0.949)$, which came from Theorem 1 , Corollaries 1 and Theorem 2, respectively. Hence, the datum shows the correction of the final sentence in Remark 7 .

\section{Conclusion}

In this paper, the finite-time synchronization issue between two complex dynamical networks is discussed. Based on the Lyapunov stability, the periodically intermittent control schemes are proposed to synchronize such network in finite time by combining adaptive control method and feedback control technique, respectively. Some sufficient conditions ensuring the global finite-time stability of the synchronization process are derived. Numerical examples are presented to verify the effectiveness of the proposed synchronization criteria 
finally.

\section{Acknowledgments.}

The authors would like to thank the editor and the anonymous reviewers for their valuable comments and constructive suggestions.

This research is supported by the National Natural Science Foundation of China (Grant No.61174216, No. 61273183, No.6134028 and No. 61374085) and the Doctoral Scientific Foundation of China Three Gorges University (Grant No. 0620120132).

\section{References}

[1] S.H. Strogatz, Exploring complex networks, Nature 410 (2001) 268-276.

[2] M.E.J. Newman, The structure and function of complex networks, SIAM Rev. 45(2) (2003) 167-256.

[3] S. Boccaletti, V. Latora, Y. Moreno, M. Chavez, D.-U. Hwang, Complex networks: Structure and dynamics, Phys. Rep. 424 (2006) 175-308.

[4] J.A.K. Suykens, G.V. Osipov, Introduction to focus issue: synchronization in complex networks, Chaos 18 (2008) 037101.

[5] Tae H. Lee, Ju H. Park, H.Y. Jung, S.M. Lee, Synchronization of a delayed complex dynamical network with free coupling matrix, Nonlinear Dyn. 69 (2012) 1081-1090.

[6] Tae H. Lee, Zheng-Guang Wu, Ju H. Park, Synchronization of a complex dynamical network with coupling time-varying delays via sampled-data control, Applied Mathematics and Computation 219 (2012) 1354-1366.

[7] Tae H. Lee, D.H. Ji, Ju H. Park, H.Y. Jung, Decentralized guaranteed cost dynamic control for synchronization of a complex dynamical network with randomly switching topology, Applied Mathematics and Computation 219 (2012) 996-1010.

[8] H. Yu, X. Xia, Adaptive consensus of muti-agents in networks with jointly connected topologies, Automatica 48 (2012) 1783-1790.

[9] H. Yu, Y. Shen, X. Xia, Adaptive finite-time consensus in muti-agent networks, System and Control Letters 62 (2013) 880-889.

[10] J. Lu, J. Cao, Adaptive synchronization of uncertain dynamical networks with delayed coupling, Nonlinear Dyn 53 (2008) 107-115.

[11] C. Zhou, J. Kurths, Dynamical weights and enhanced synchronization in adaptive complex networks, Physical Review Letters 96 (2006) 164102.

[12] P. DeLellis, M. di Bernardo, F. Garofalo, M. Porfiri, Evolution of complex networks via edge snapping, IEEE Transactions on Circuits and System I 57(8) (2012) 2153-2158.

[13] W. Yu, P. Delellis, G. Chen, M. di Bernardo, J. Kurths, Distributed adaptive control of synchronization in complex networks, IEEE Transactions on Automatic. Control 57(8) (2012) 2153-2158.

[14] P. Delellis, M. di Bernardo, F. Garofalo, Novel decentralized adaptive strategies for the synchronization of complex networks, Automatica 45(5) (2009) 1312-1318.

[15] U.E. Vincent, R. Guo, Finite-time synchronization for a class of chaotic and hyperchaotic systems via adaptive feedback controller, Phys. Lett. A 375 (2011) 2322-2326.

[16] Ju H. Park, Synchronization of cellular neural networks of neural type via dynamic feedback controller, Chaos Solitons Fractals 42 (2009) 1299-1304. 
[17] Y. Xu, W. Zhou, J. Fang, H. Lu, Structure identification and adaptive synchronization of uncertain general complex dynamical networks, Phys. Lett. A 374 (2009) 272-278.

[18] R. Guo, U.E. Vincent, Finite-time stabilization of chaotic systems via single input, Phys. Lett. A 375 (2010) 119-124.

[19] Y. Shen, Y. Huang, Jason. Gu, Global finite-time observers for lipschitz nonlinear systems, IEEE Trans. Autom. Control 56 (2011) 418-424.

[20] Y. Shen, Y. Huang, Uniformly observable and globally lipschitzian nonlinear system admit global finite-time observers, IEEE Trans. Autom. Control 54 (2009) 2621-2625.

[21] Q. Song, J. Cao, On pinning synchronization of directed and undirected complex dynamical networks, IEEE Trans. Circcuits Syst. I, 57 (2010) 672-680.

[22] K. Yuan, G. Feng, J. Cao, Pinning control of coupled networks with time delay, The Open Electr. and Eng. 6 (2012):14-20.

[23] P. DeLellis, M. di Bernardo, M. Porfiri, Pinning control of complex networks via edge snapping, Chaos 21(2011) 033119.

[24] X. Yang, J. Cao, J. Lu, Synchronization of delayed complex dynamical networks with impulsive and stochastic effects, Nonlinear Analysis: RWA 12 (2011) 2252-2266.

[25] Q. Zhang, J. Lu, J. Zhao, Impulsive synchronization of general continuous and discrete-time complex dynamical networks, Commun Nonlinear Sci Numer Simulat 15 (2010) 1063-1070.

[26] M. Sun, C. Zeng, Y. Tao, L. Tian, Adaptive-impulsive synchronization in drive-response networks of continuous systems and its application, Phys. Lett. A 373 (2009) 3041-3046.

[27] S. Zheng, G. Dong, Q. Bi, Impulsive synchronization of complex networks with non-delayed and delayed coupling, Phys. Lett. A 373 (2009) 4255-4259.

[28] S. Cai, J. Zhou, L. Xiang, Z. Liu, Robust impulsive synchronization of complex delayed networks, Phys. Lett. A 372 (2008) 4990-4995.

[29] W. Xia, J. Cao, Pinning synchronization of delayed dynamical networks via periodically intermittent control, Chaos 19 (2009) 013120.

[30] S. Cai, Z. Liu, F. Xu, J. Shen, Periodically intermittent controlling complex dynamcial networks with time-varying delays to a desired orbit, Phys. Lett. A 373 (2009) 3846-3854.

[31] S. Cai, J. Hao, Q. He, Z. Liu, Exponential synchronization of complex delayed dynamical networks via pinning periodically intermittent control, Phys. Lett. A 375 (2011) 1965-1971.

[32] Y. Wang, J. Hao, Z. Zuo, A new method for exponential synchronization of chaotic delayed systems via intermittent control, Phys. Lett. A 374 (2010) 2024-2029.

[33] J. Mei, M. Jiang, W. Xu, B. Wang, Finite-time synchronization control of complex dynamical networks with time delay, Commun Nonlinear Sci Numer Simulat 18(2013) 2462-2478.

[34] H. Zhu, B. Cui, Stabilization and synchronization of chaotic systems via intermittent control, Commun Nonlinear Sci Numer Simulat 15 (2010) 3577-3586.

[35] S. Cai, J. Hao, Z. Liu, Exponential synchronization of chaotic systems with time-varying delays and parameter mismatches via intermittent control, Chaos 21 (2011): 023112.

[36] J Mei, M. Jiang, B. Wang, Q. Liu, W. Xu, T. Liao, Exponential p-synchronization of nonautonomous Cohen-Grossberg neural networks with reaction-diffusion terms via periodically intermittent control, Neural Process Lett., DOI: 10.1007/s11063-013-9313-x.

[37] P. DeLellis, M. di Bernardo, F. Garofalo, D. Liuzza, Analysis and stability of consensus in networked control systems, Applied Mathematics and Computation, Special Issue In Honor of George Leitmann, 217(3) (2010) 988-1000. 
[38] C. Li, G. Feng, X. Liao, Stabilization of Nonlinear Systems via Periodically Intermittent Control, IEEE Trans. Circuits Syst. II 54 (2007) 1019-1023.

[39] S. Cai, Q. He, J. Hao, Z. Liu, Exponential synchronization of complex networks with nonidentical time-delayed dynamcial nodes, Phys. Lett. A 374 (2010) 2539-2550.

[40] C. Hu, J. Yu, H. Jiang, Z. Teng, Exponential synchronization of complex networks with finite distributed delays coupling, IEEE Trans. Neural Networks 22 (2007) 1999-2010.

[41] Y. Tang, Terminal sliding mode control for rigid robots, Automatica 34 (1998) 51-56.

[42] X. Huang, W. Lin, B. Yang, Global finite-time synchronization of a class of uncertain nonlinear systems, Automatica 41(2005) 881-888.

[43] H. Wang, Z. Han, Q. Xie, W. Zhang, Finite-time chaos control via nonsingular terminal sliding mode control, Commun Nonlinear Sci Numer Simulat 14 (2009) 2728-2733.

[44] P. DeLellis, M. di Bernardo, G. Russo, On QUAD, lipschitz and contracting vector fields for consensus and synchronization of networks, IEEE Transactions on Circuits and Systems I 58(3) (2011) 576-583.

[45] M. Pourmahmood, S. Khanmohammadi, G. Alizadeh, Finite-time synchronization of two different chaotic systems with uncertain parameters via sliding mode technique, Appl. Math. Modelling 35 (2011) 3080-3091.

[46] R. Luo, Y. Wang, Finite-time stochastic combination synchronization of three different chaotic systems and its application in secure communication, Chaos 22 (2012) 023109.

[47] J. Mei, M. Jiang, J. Wang, Finite-time structure identification and synchronization of drive-response systems with uncertain parameter, Commun Nonlinear Sci Numer Simulat 18 (2013) 999-1015.

[48] J. Mei, M. Jiang, B. Wang, B. Long, Finite-time parameter identification and adaptive synchronization between two chaotic neural networks, Journal of The Franklin Institute 350 (2013) 1617-1633. 